\title{
Semi-supervised human resource scheduling based on deep presentation in the cloud
}

\author{
Yuanmo Lin ${ }^{1}$, Xinmei Wang ${ }^{2}$ and Rongbin $\mathrm{Xu}^{1,3^{*}}$
}

\begin{abstract}
Traditional competitive human resource allocation is no longer suitable for contemporary requirements. To improve the effectiveness of human resource management and the degree of matching between jobs and staffs, we propose a novel auto-encoder neural network-based method in the cloud environment, which is a semi-automatic manner in the business process of human resource allocation. The proposed method is based on deep learning architecture by using appropriate cloud resources and takes into account the similarities and deep presentation between staff modules. The construction between human resource network is combined with a priori information about the human resource and set up by the evaluation index system of human resource planning. Our proposed method enables the modeling of semi-automatic human resource allocation process and can be used to facilitate optimized human resource allocation. Experimental results show that our method can bring significant improvements to personnel position matching and effectively enhance the efficiency of human resource allocation based on the cloud environment.
\end{abstract}

Keywords: Semi-supervised learning, Cloud computing, Human resource allocation, Staff relationship, Neural network

\section{Introduction}

Human resource is an important factor that affects the survival and development of an enterprise in contemporary competitive business environments [1]. The allocation of human resources aims to improve the matching degree between personnel and positions. The human resource allocation directly affects the overall utilization efficiency of other resources in an enterprise [2]. Effective allocation of human resources is a key factor that determines whether an enterprise can sustain, stabilize, and develop rapidly. Accurate prediction of the required human resource for modern enterprises can help the enterprises find out whether the personnel positions are insufficient or too many, which will provide accurate guidance for rational allocation of human resources that

*Correspondence: xurb_910@126.com

${ }^{1}$ College of Information Engineering, Putian University, Putian, China

${ }^{3}$ Engineering Research Center of Big Data Application in Private Health

Medicine, Fujian Province University, Putian, China

Full list of author information is available at the end of the article has far-reaching significance for the sustainable development of enterprises [3]. By the convenience of using cloud computing resources, the proper allocation of enterprise human resources will contribute to the formation of meaningful economic activities and, thus, greatly improve the productivity of human resources [4]. The ultimate goal of enterprise human resource allocation is to improve the overall operating efficiency and create more economic and social benefits for enterprises. Therefore, nowadays, various human resource information systems based on cloud environment mainly focus on the development of decision support function and the research on how to use existing human resource data to further optimize the matching relationship between internal employees and post requirements. Such a data-driven approach can not only solve the practical problems in business operation, but also provide scientific decision support for optimized human resource allocation [5-7]. 
In general, cloud-based staff assignment will be set up during the business process establishment phase, where it gives better control over the resources required to perform an activity [8]. In the execution phase, business process management can automatically integrate resources into the corresponding work and redistribute them to the corresponding execution roles [9]. We can also use simple mechanisms such as sequence length or round-robin for resource allocation. However, in many real-world applications, such simple execution-phase allocation mechanisms do not work well when allocating staff and resources. For example, in order to ensure product quality [10], a study of an engineering design process in a manufacturing enterprise reveals that a typical component design is typically assigned manually by the business process management initiator [11]. According to the current research status, these manual personnel assignment jobs often occur in manufacturing enterprises, especially in some important business processing tasks.

Actually, human resource allocation involves two aspects: personnel and positions. for the essence that it matches the human resources with positions. For decades, enterprises and academia have realized the importance of rational human resource allocation for an organization and have been committed to studying these related fields [12]. And the development of cloud computing technology brings opportunities for human resource scheduling. Therefore, the goal of all human resource development theories is to select the right person for the right place by cloud resources. The rational allocation of human resources is influenced by many factors such as scale, economic strength, culture, and the external environment of the enterprise. At the same time, most of these factors are nonlinear [13-15]. However, some traditional analysis methods such as multiple linear regression (LR) and analytic hierarchy process (AHP) are linear analysis [16]. However, human resource allocation data are complicated, and the obvious disadvantages of these traditional methods are strong subjectivity, low accuracy, and weak capability to reflect the actual situation. In contrast to the traditional linear methods, artificial neural networks (ANNs) have unique advantages in dealing with nonlinear problems based on cloud environment. ANNs have strong self-learning ability, self-organizing characteristics, extensive adaptability, fault tolerance, and predictability, which help to deal various complex issues in human resource allocation. In this paper, we adopt stack auto-encoder neural network to predict the required human resource and fully consider the characteristics of similarity between different staff, which combines the prior information of position fitness constraint to effectively realize the reasonable allocation of semi-supervised human resources.

The major contributions of this paper are listed as follows:
- We propose a new method of human resource scheduling method with sparse constraint, which can efficiently represent the characteristics of human resource in the cloud environment.

- We propose a new deep neuro-network structure with four auto-encoder layers to get the human resource feature, which can construct a relationship between features and promote the prediction of scheduling.

- We propose a novel method to calculate the post fitness constraint. Assuming that both person $i$ and person $j$ are suitable for the same post, so there must be some similar attributes between person $i$ and $j$. Our new post fitness constraint can achieve good classification result.

The remainder of this paper is organized as follows. Section 2 introduces the related work. In Section 3, we propose a novel human resource scheduling strategy in the cloud. In Section 4, we conduct extensive experiments to compare our algorithm with the other two state-of-theart prediction methods. We make a conclusion and point out our future work in Section 5.

\section{Related work}

For human resource allocation, one of the core business processes is to identify and recruit people with corresponding skills, abilities, knowledge, and experience to fill the posts in enterprise organization [17-19]. Although there are many human resource scheduling strategies and mechanisms in the field of human resource scheduling, there are still some drawbacks in the existing research work. Liu et al. [20] introduced BP neural network to conduct some comprehensive evaluation and analysis on performance evaluation of enterprise human resource management; this method provides a scientific and effective human resource evaluation system for enterprises. Feng et al. [21] built an effective human resource allocation model by summarizing the modularity, timeliness, and reciprocity of human resource allocation, which utilizes customer value and human resource core competence and takes trust mechanism as the links. Sliva et al. [22] proposed a knowledge-based method to match the job seekers. This method collected the data from applicants through web interface and matched suitable jobs through a web-based human resource management system model by the network information server. However, they have not considered the sparse constraint in their scheduling strategies.

Li et al. [23] analyzed the matching relationship between human resource management practice and organizational situation based on BP neural network model, which established a prediction model and received a good prediction effect. Wang et al. [15] studied a large number of 
influencing factors related to human resources, which proved the effect of flexible degree of personnel resources on the scheduling result. Zhang et al. [24] described human resources by attributes such as skills, salary, and degree of dedication to projects, so as to establish scheduling constraints and realize human resource scheduling by genetic algorithm. Liu et al. [25] provided an up-todate overview on four deep learning architectures, which highlights the applications of deep learning techniques on some selected areas. Similarly, the authors in [23] constructed a deep BP neural network in conjunction with specific old-age care scenarios, which was trained as a whole structure by using instance monitoring data. They all represent the characteristics of human resource. However, they have not fully constructed the real relationship between input features and promoted scheduling methods.

Although there are already many personnel allocation strategies and mechanisms in the field of business process management research, these research efforts are less likely to be carried out at execution time to flexibly recommend resources based on collaborative behavior relationships $[26,27]$. In work [28-31], researchers found that business process management history information could be used to improve the efficiency of cloud resource allocation during business process management execution. Russell et al. [32, 33] introduced a new pattern that integrated work items and scheduling resources based on the previous execution history of cloud resources. Xu et al. and Priya et al. [34, 35] put forward the event of logging method to study the allocation law, which made the resource allocation work in the execution phase more effective.

The abovementioned methods have made great achievements in human resource scheduling by cloud environment. However, most of them do not take into account the nonlinear influencing factors in the human resource allocation process as well as the specific relationship between personnel that results static distribution of cloud resources. Moreover, they do not provide enough descriptions of human resource characteristics in the post allocation process. In what follows, we will propose a new deep nonlinear reconstruction model of human resources to solve the deficiencies in the traditional human resource allocation process based on cloud environment, which can construct the relationship between features and prediction results.

\section{Human resource scheduling based on deep representation in cloud environment}

The allocation of enterprise human resources in cloud environment should meet the reasonable matching of personnel and specific positions as shown in Fig. 1. In the distribution process, the people who are suitable for the same post often have certain similarities. In order to better predict unallocated employees for the most suitable job, we will use different attributes to construct the human resource network based on personnel relationship module and put forward a novel nonlinear reconstruction model about human resource scheduling in the cloud.

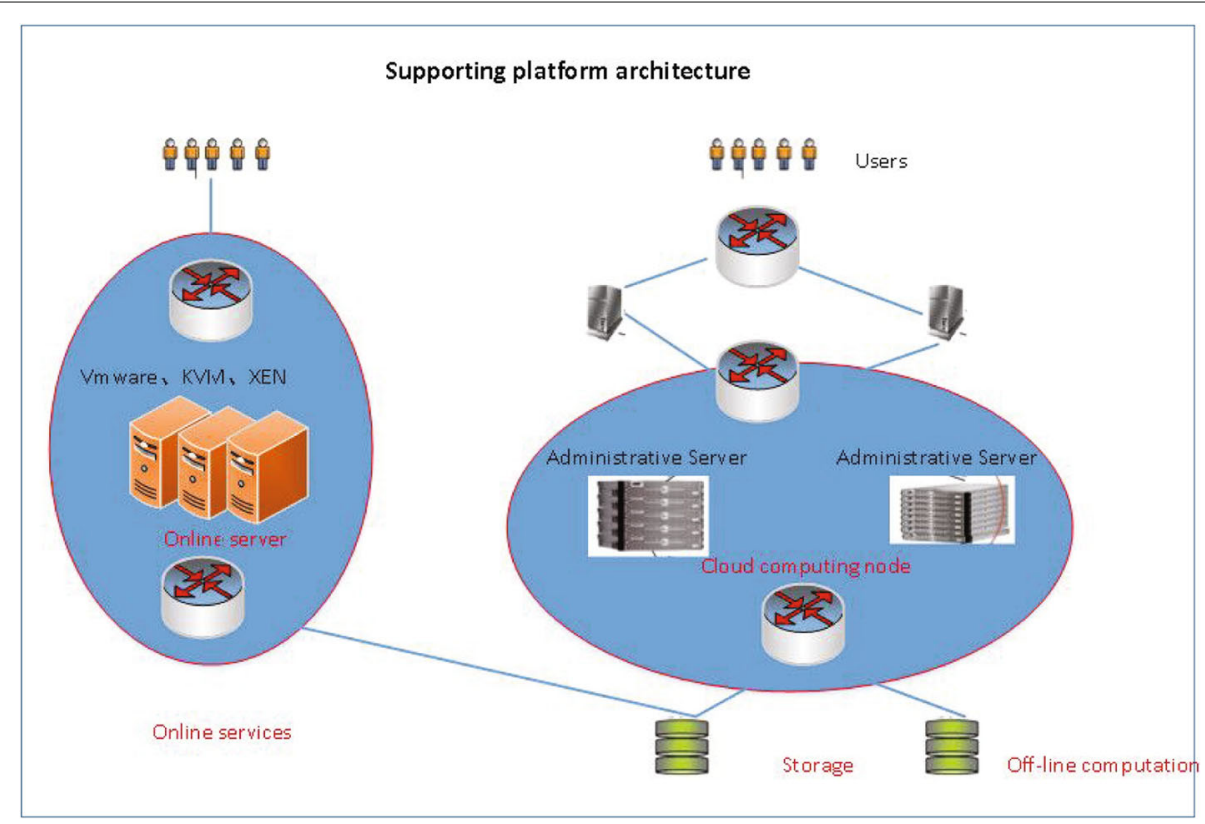

Fig. 1 The architecture of human resource scheduling in the cloud 
The depth of human resource allocation will make the matching process more reasonable so that all the tasks can be completed in time.

\subsection{Construction of human resource network}

In the process of human cloud resource allocation, each person has its specific attributes and outstanding merits according to the working characteristics of different human resources. For example, the attributes of a person are $X=$ "proficient in Java," "strong logical thinking,,.., "team work." These attributes show that the person is well versed in Java programming and has good experience in teamwork process. This indicates that the employee is very suitable for the above work. Then this employee will be more reliable for the post of Java development. The attributes that the persons require for the same position should be similar. Therefore, a specific human resource network can be constructed based on the attributes of each person.

If $N$ job seekers are given and $M$ attributes are set, a human resource network $G=(V, E, L)$ can be formed. $V=\left\{v_{1}, v_{2}, \ldots, v_{N}\right\}$ is the vertex set, which represents $N$ job seekers. $E=\left\{e_{i j}\right\}$ is the set of edges connecting two vertices in $V . Y=\left\{Y_{L A}, Y_{U L}\right\}$ is the class standard of personnel, which is the matched post. The labels of staffs assigned to a post are $Y_{L A}=\left\{Y_{1}, \ldots, Y_{L}\right\}$, then the personnel category of unassigned posts $Y_{U L}=\left\{Y_{(L+1)}, Y_{N}\right\}$ can be obtained. The adjacency matrix of human resource network $G$ can be constructed from human resource relationship, which is a non-negative symmetric matrix $A=$ $\left[a_{i j}\right] \in R_{+}^{N \times N}$. If there are edges between vertices $i$ and $j$, then $a_{i j}=1$, else $a_{i j}=0$. For all $1 \leq i \leq N$, we have $a_{i i}=0$. Now we can introduce a symmetric matrix $W=$ $\left[w_{i j}\right] \in R_{+}^{N \times N}$ to depict the similarity of human resource in the personnel network when $a \in R^{N}$.

$$
w_{i j}=\exp \left(-\sum_{k=1}^{N} \frac{\left(a_{i k}-a_{j k}\right)^{2}}{\sigma_{k}^{2}}\right)
$$

In Eq.(1), $a_{i k}$ is the $k$ th element of adjacency matrix vector $a_{i}$, and $\sigma$ is the scale of the control parameter for each dimension.
Furthermore, considering the relation between different staff who are suitable for the same position, we introduce a personnel relationship matrix $M=\left[m_{i j}\right] \in R^{N \times N}$

$$
m_{i j}=w_{i j}-\frac{\sqrt{k_{i}^{2}+k_{j}^{2}}}{\sum_{i, j} w_{i j}}
$$

where $m$ is a further optimization of $w$ that can be used to better capture personnel relationship of human resource. $k_{i}$ is the degree of staff $i$.

\subsection{Deep nonlinear distribution distance reconstruction model for human resource allocation}

Based on the nonlinear characteristics of human resource relation network in cloud environment, a novel model is adopted to deal with the problem of human resource allocation. An important component module that is named auto-encoder is introduced in this model. Auto-encoder is a special neural network used to learn a new representation of the original data. The composition of auto-encoder is shown in Fig. 2.

The data of $x$ are input into the auto-encoder structure. They will be encoded by the encoder module and be decoded by the decoder module. Then the decoded data will be compared with the input data through the reconstruction process. Based on the auto-encoder structure, we can intend to minimize the error between input and output data.

As the input of auto-encoder by the personnel relationship matrix $M$, the encoded personnel relationship matrix $M$ will be mapped into a low dimensional embedding $H=$ $\left[h_{i j}\right] \in R^{d \times N}$, where $d<N$. So we can get the following formula,

$$
h_{i}=f\left(m_{i}\right)=s\left(W_{H} m_{i}+d_{H}\right)
$$

where $W_{H} \in R^{d \times N}, d_{H} \in R^{d \times 1}$ is the weight of coding layer and bias vector and $s($.$) is a nonlinear mapping func-$ tion, that is the Softmax function $s_{\text {softmax }}(x)=\frac{e^{x}}{\sum_{i} e^{x_{i}}}$. This function takes the vector as input and normalizes it into a probability distribution consisting of input vector's probabilities proportional to the exponentials. After decoding,

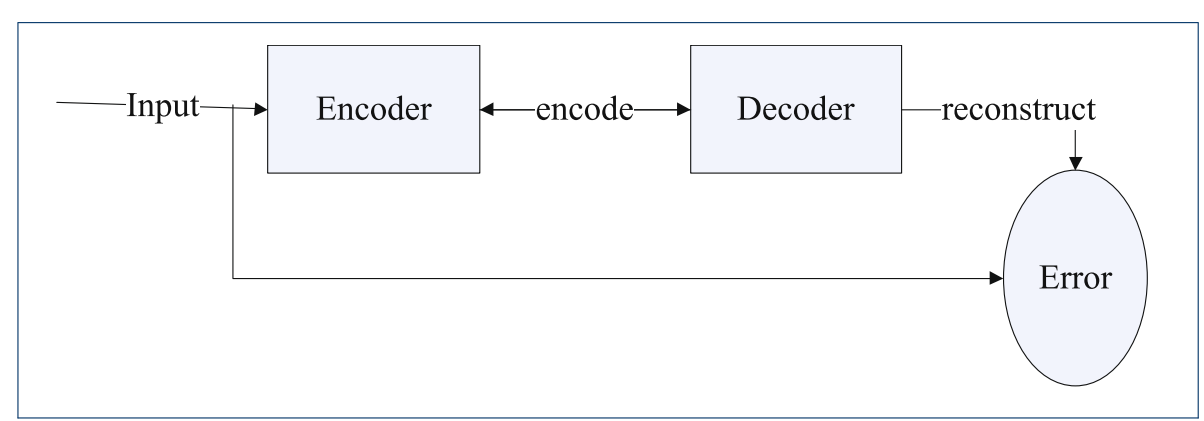

Fig. 2 The structure of auto-encoder 
the hidden layer representation $H$ is reflected to the original data space to yield a reconstruction of the original data.

$$
o_{i}=g\left(h_{i}\right)=s\left(W_{O} h_{i}+d_{O}\right)
$$

where $W_{O} \in R^{N \times d}, d_{O} \in R^{N \times 1}$ is the weight of decoding layer and bias vector. Auto-encoder is mainly to study in the condition of a parameter $\theta=\left\{W_{H}, d_{H}, W_{O}, d_{O}\right\}$, which will reconstruct the original data to minimize the raw data $M$ and the reconstruct the deviation between data $O$. So that a low dimensional nonlinear representation of $H$ can be obtained.

$$
\begin{aligned}
\text { cost } & =\underset{\theta}{\arg \min } L_{\theta}(M, O)=\underset{\theta}{\arg \min }|| M-O \|^{2} \\
& =\underset{\theta}{\arg \min } \sum_{i=1}^{N}\left(m_{i}-o_{i}\right)^{2}
\end{aligned}
$$

In addition, a sparsity constraint is added to the hidden layer by imposing a restriction condition on the neural network of auto-encoder.

$$
\sum_{j=1}^{P} K L\left(\rho \| \rho_{j}\right)=\sum_{j=1}^{P} \rho \log \frac{\rho}{\rho_{j}}+(1-\rho) \log \frac{1-\rho}{1-\rho_{j}}
$$

where $\rho$ is the sparsity parameter, $P$ is the number of neurons in the hidden layer, and $\rho_{j}=\frac{1}{N} \sum_{i} h_{i}$ represents the average activity of hidden neurons.

Thus, the total cost function of neural network for autoencoder can be obtained.

$$
\operatorname{COST}=\operatorname{cost}+\sum_{j=1}^{P} \mathrm{KL}\left(\rho \| \rho_{j}\right)
$$

Here, cost is the result of Formula 5, which is the reconstruction between $M$ and $O$. COST is the summation between cost and KL distance.
To better achieve human resource scheduling in the cloud, a novel deep structure is presented that can improve the match between personnel and posts. As shown in Fig. 3, this structure is a layer by layer training network which applies four auto-encoder stacks to form a stack auto-encoder.

For a deep auto-encoder network, we train the first auto-encoder by reconstructing raw data in human relation matrix $B$ and get a hidden layer representation $H^{1} \in$ $\left.R^{(} N \times t_{1}\right)$. Then $H^{1}$ can be served as the input of the next auto-encoder. And the rest layers can be done in the same manner. So the fourth auto-encoder will get a new representation $H^{4} \in R^{N \times t_{4}}$ by reconstructing the output of the third auto-encoder.

Assuming that both person $i$ and person $j$ are suitable for the same post, there must be some similar attributes between persons $i$ and $j$, which can be regarded as a priori information for human resource scheduling. Then a post fitness matrix $S=\left[s_{i j}\right] \in R_{+}^{N \times N}$ can be defined to represent the adaptability of person $i$ and person $j$ for a certain post, the threshold range is from 0.1 to 1 . Meanwhile, Euclidean distance is used to measure the similarity $D\left(h_{i}, h_{j}\right)$ of the hidden layer after training. Therefore, the post fitness constraint can be written as the following formula.

$$
\begin{aligned}
& R(S, H)=\frac{1}{2} \sum_{i=1}^{N} \sum_{j=1}^{N} s_{i j} D\left(h_{i}, h_{j}\right) \\
& =\frac{1}{2} \sum_{i=1}^{N} \sum_{j=1}^{N} s_{i j}\left\|h_{i}-h_{j}\right\|_{1}+\|S\|_{1} \\
& =\operatorname{Tr}\left(H^{T} D H\right)-\operatorname{Tr}\left(H^{T} S H\right) \\
& =\operatorname{Tr}\left(H^{T} L H\right)
\end{aligned}
$$

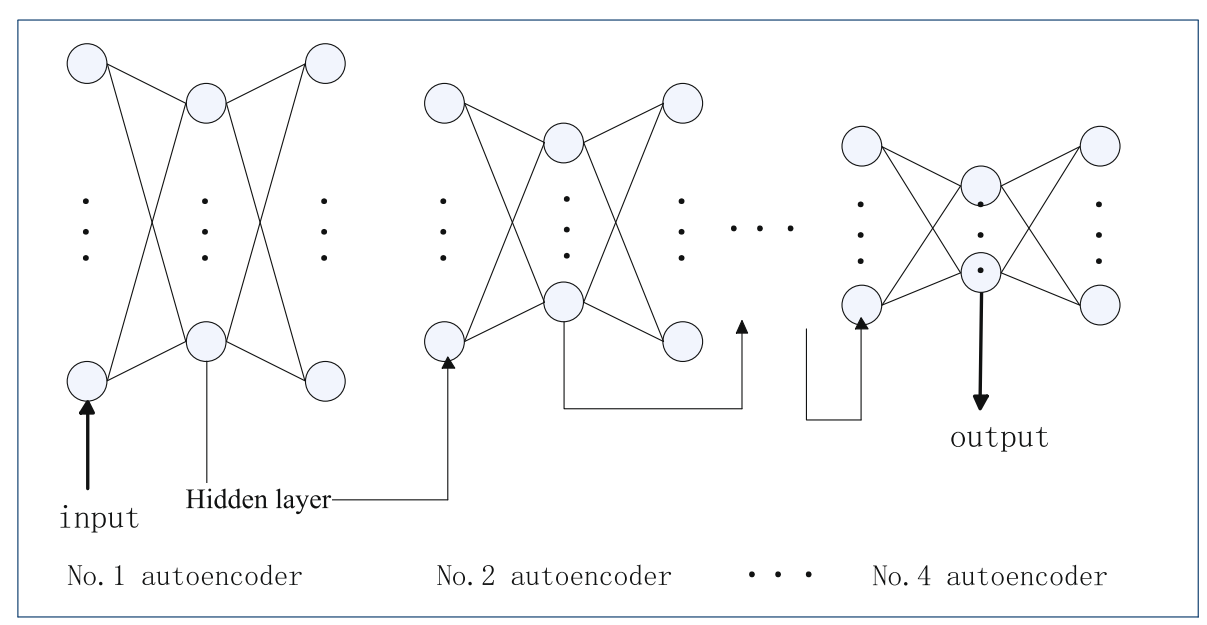

Fig. 3 The structure of stack auto-encoder 
where $\operatorname{Tr}($.$) is the trace of the matrix and diagonal matrix$ $\left.D=\left[d_{i j}\right] \in R_{+}^{(} N \times N\right)$ is the summation of per line in matrix $S . d_{i i}=\sum_{j=1}^{N} s_{i j}, L=D-S$ is the Laplacian matrix of $S$. It follows that the new loss function can be obtained.

$$
\mathrm{COST}^{*}=\mathrm{COST}+\lambda \operatorname{Tr}\left(H L H^{T}\right)
$$

where $\lambda$ is the parameter between the regulatory reconstruction deviations and constraints.

Our proposed method uses the back propagation algorithm of stochastic gradient descent to optimize the autoencoder. The parameter $\theta=\left\{W_{H}, d_{H}, W_{O}, d_{O}\right\}$ is updated during each iteration and the network will be trained continuously to effectively realize the personnel position matching process.

\section{Experiment results and analysis}

The dataset of a given automobile manufacturing enterprise is used to test the performance of the human resource scheduling method proposed by this paper in the cloud environment this paper. This dataset is divided into 10 subsets with the same size and no mutual inclusion. Then deep nonlinear reconstruction model for human resource is applied to train the first 9 subsets and the other subset is used as test data. The average value of 10 results is used as the accuracy to estimate the precision of our algorithm. According to the similarity between personnel in training dataset, cross-validation is used to evaluate the accuracy of matching between personnel and positions in this model.

\subsection{Experiment dataset}

The dataset applied in this paper is the relevant data of an automobile manufacturing enterprise, from which 2000 people are randomly selected as the experimental data. As the deep nonlinear reconstruction model for human resource allocation, the stacked auto-encoder network consists of four auto-encoders. The layer allocation of experimental data is set as [2000-512-256-128-64].

That is the first auto-encoder is 2000-512-2000 and the second one is 512-256-512. The third auto-encoder is 256-128-256 and the fourth one is 128-64-128. All the auto-encoders are individually trained. Then the trained hidden layer representation is selected from the four autoencoders for clustering. Here, $k$-means is adopted as the clustering method to obtain the class standard information of personnel.

\subsection{Index of performance evaluation}

In this paper, Accuracy and AUC are adopted as standard evaluation metrics for human resource allocation.

$$
\text { Accuracy }=\frac{\mathrm{TP}+\mathrm{TN}}{\mathrm{TP}+\mathrm{TN}+\mathrm{FP}+\mathrm{FN}}
$$

Table 1 Accuracy of the proposed algorithm under different numbers of personnel

\begin{tabular}{llll}
\hline $\begin{array}{l}\text { Number of } \\
\text { personnel }\end{array}$ & DNR 2 algorithm & DNREE algorithm & $\begin{array}{l}\text { The proposed } \\
\text { algorithm }\end{array}$ \\
\hline 340 & 0.87 & 0.89 & $\mathbf{0 . 8 9}$ \\
620 & 0.87 & 0.81 & $\mathbf{0 . 8 9}$ \\
680 & 0.87 & 0.81 & $\mathbf{0 . 9 1}$ \\
1050 & 0.82 & 0.75 & $\mathbf{0 . 9 2}$ \\
1150 & 0.92 & 0.91 & $\mathbf{0 . 9 4}$ \\
1490 & 0.87 & 0.85 & $\mathbf{0 . 9 5}$ \\
\hline
\end{tabular}

where TP represents the number of correctly divided into positive examples, FP represents the number of incorrectly divided into positive examples, $\mathrm{TN}$ represents the number of correctly divided into negative examples, and FN represents the number of incorrectly divided into negative examples. The evaluation method AUC (area under the curve of receiver operating characteristic) is the standard metric for judging the advantages and disadvantages of prediction model.

$$
\mathrm{AUC}=\frac{\sum_{i \in \text { positiveClass } \operatorname{rank}_{i}-\frac{M(1+M)}{2}}}{M \times N}
$$

\subsection{Experiment results and discussion}

We randomly select different numbers of personnel data in the dataset. Table 1 shows the experiment results under different numbers of selected personnel. For comparison purposes, two conventional methods are chosen as the baseline. One is the $\mathrm{DNR}_{L 2}$ [36] algorithm, which applies L2 normal form to error loss function; the other is the $\mathrm{DNR}_{\mathrm{CE}}$ [37] algorithm, which applies sigmoid-cross entropy to error function. As shown in Tables 1 and 2, the proposed method in this paper is superior to the DNR $\left(\mathrm{DNR}_{L 2}\right)$ algorithm and the DNR (DNR $\mathrm{CE}$ ) algorithm under the condition of different numbers of personnel. Because our proposed algorithm fully considers the related attributes between personnel and adopts the position fitness constraint prior information after personnel similarity processing, it not only improves the efficiency

Table 2 AUC of the proposed algorithm under different numbers of personnel

\begin{tabular}{llll}
\hline $\begin{array}{l}\text { Number of } \\
\text { personnel }\end{array}$ & DNR $R_{L 2}$ algorithm & DNR $R_{C E}$ algorithm & $\begin{array}{l}\text { The proposed } \\
\text { algorithm }\end{array}$ \\
\hline 340 & 0.91 & 0.91 & $\mathbf{0 . 9 6}$ \\
620 & 0.90 & 0.87 & $\mathbf{0 . 9 2}$ \\
680 & 0.69 & 0.79 & $\mathbf{0 . 9 0}$ \\
1050 & 0.74 & 0.76 & $\mathbf{0 . 9 0}$ \\
1150 & 0.93 & 0.92 & $\mathbf{0 . 9 8}$ \\
1490 & 0.89 & 0.90 & $\mathbf{0 . 9 8}$ \\
\hline
\end{tabular}




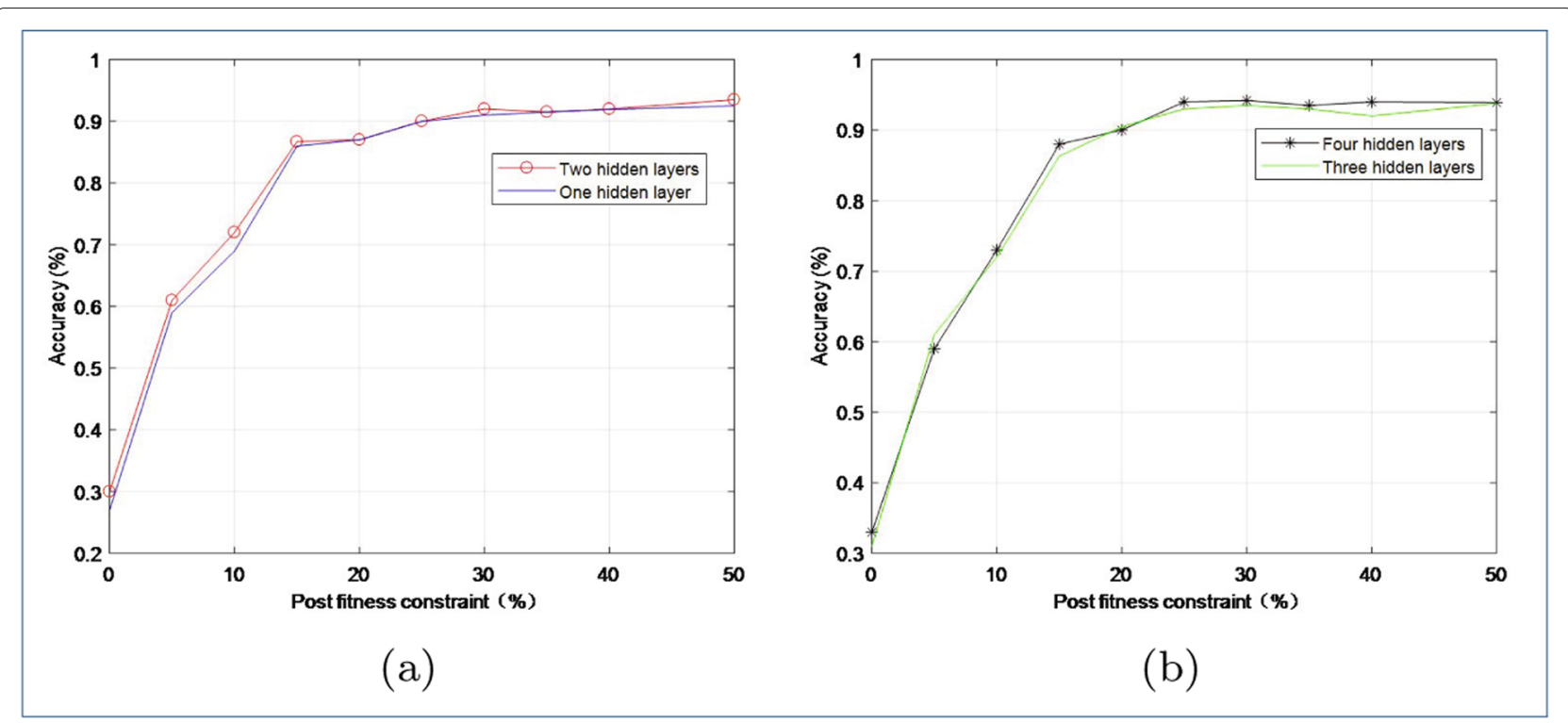

Fig. 4 Training results of stack auto-encoder hidden layer by post fitness constraint

but also avoids the complexity of manually matching personnel positions. Therefore, the allocation efficiency of human resource department has been greatly improved in the practical application.

In order to evaluate the impact of post fitness constraints on our algorithm, 2000 experimental data are stacked and trained from four hidden layers in the autoencoder. As shown in Fig. 4, it can be seen that the four hidden layers trained by the stacked auto-encoder can better reflect the significant characteristics of personnel compared with the evaluation of the other three different hidden layers so as to achieve a higher prediction ability of human resource allocation. Therefore, the deep nonlinear reconstruction model of human resource allocation based on the deep neural network proposed by this paper can achieve better degrees of job matching in cloud environment, which effectively and reasonably allocates human resources in automobile manufacturing enterprises and achieves the optimization of human resource allocation. As shown in Eq. (9) of Section 3, $\lambda$ is served as the controlling parameter between reconstruction error and different values of constraint $\lambda$ can be obtained under different value of cost function. Figure 5 shows the change in sensitivity of the cost function as the value of $\lambda$ increases. It

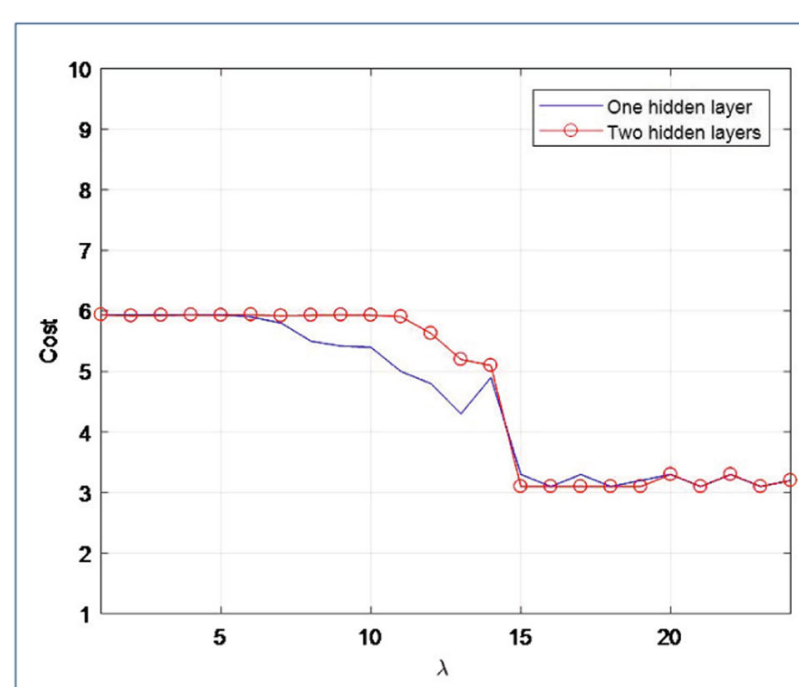

(a)

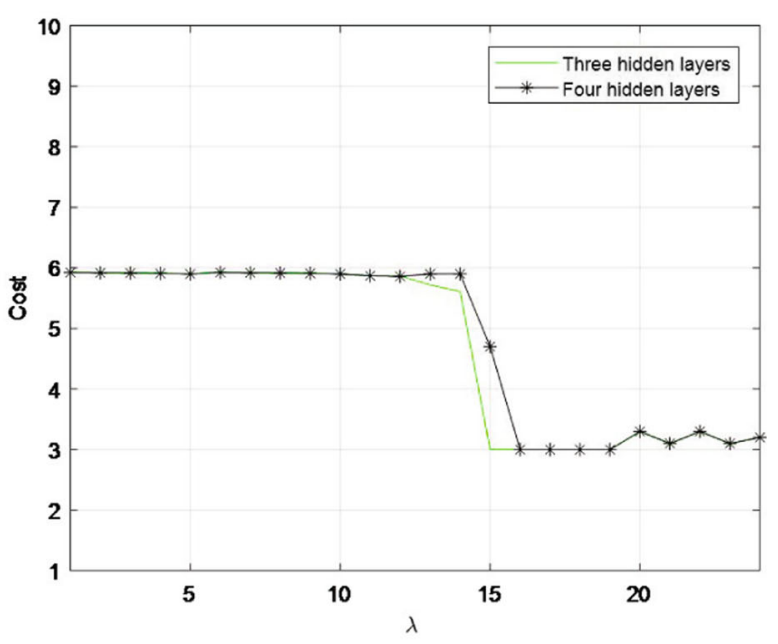

(b)

Fig. 5 Results of parameter sensitivity in stack auto-encoder cost function 
proves that the cost function of our novel method can get stable and low values by using four hidden layers, which shows the best effect in this work.

\section{Conclusion and future work}

To effectively assist enterprises to achieve the optimized allocation of human resources, this paper proposes a deep nonlinear reconstruction model for human resource allocation in cloud environment. Our model can achieve semi-supervised post-personnel matching in human resource management. The proposed method fully considers the characteristics of personnel and explores the similarity among personnel with other effective factors by cloud resources. Combining with the prior information of personnel position fitness constraints, the semi-supervised learning of personnel position matching process greatly improves the overall prediction ability based on the relationship module between personnel. The proposed algorithm is tested on a dataset of automobile manufacturing enterprise. Experimental results show that the model proposed in this paper can achieve remarkable results in personnel position matching, which effectively improves the efficiency of human resource allocation.

Our future work will try to apply the method proposed in this paper to the study of personnel arrangement process in projects for cloud resource allocation, considering that the working ability of personnel will directly affect the efficiency of project completion.

\section{Abbreviations}

LR: Linear regression; AHR: Analytic hierarchy process; ANNs: Artificial neural networks; BP: Back propagation

\section{Acknowledgements \\ We gratefully acknowledge the anonymous reviewers for taking time reviewing our manuscript.}

\section{Authors' contributions}

Lin and Xu conceived and designed the study. Xinmei Wang performed the simulations. Lin and Xu wrote the paper. All authors reviewed and edited the manuscript. The authors read and approved the final manuscript.

\section{Funding}

The research is supported by the Young and Middle-aged Teachers Education Research Project of Fujian Province (JAT170504), Putian Technology Bureau (2019GP002, 2019GP0011), Natural Science of Anhui (1808085MF199, KJ2018A0016), and Funds of Putian University $(2019020,2019021)$.

\section{Availability of data and materials}

We declared that materials described in the manuscript, including all relevant raw data, will be freely available to any scientist wishing to use them for non-commercial purposes, without breaching participant confidentiality.

\section{Competing interests}

The authors declare that they have no competing interests.

\section{Author details}

${ }^{1}$ College of Information Engineering, Putian University, Putian, China. ${ }^{2}$ School of Computer Science and Technology, Anhui University, Hefei, China.
${ }^{3}$ Engineering Research Center of Big Data Application in Private Health Medicine, Fujian Province University, Putian, China.

Received: 9 January 2020 Accepted: 6 March 2020

Published online: 03 April 2020

References

1. C. Boon, R. Eckardt, D. P. Lepak, P. Boselie, Integrating strategic human capital and strategic human resource management. Int. J. Hum. Resour. Manag. 29(1), 34-67 (2018)

2. J. Shen, J. Benson, When csr is a social norm how socially responsible human resource management affects employee work behavior. J. Manag. 42(6), 1723-1746 (2016)

3. Y. Dong-Jin, W. Jiao-Jiao, L. Cheng-Fei, Approach to optimal staff assignment in workflows based on collaboration patterns. J. Soft., 3340-3354 (2018)

4. S. Jian, L. Chang-Yong, X. Jian, Trust-based multi-objectives task assignment model in cloud service system. J. Comput. Res. Dev. 55(6), 1167 (2018)

5. X. Ding, "internet plus" urban production. Comput. Integr. Manuf. Syst. 24(2), 427-443 (2018)

6. K. Imagane, K. Kanai, J. Katto, T. Tsuda, H. Nakazato, in 201815 th IEEE Annual Consumer Communications \& Networking Conference (CCNC). Performance evaluations of multimedia service function chaining in edge clouds (IEEE, 2018), pp. 1-4. https://doi.org/10.1109/ccnc.2018.8319249

7. S. Oueida, Y. Kotb, M. Aloqaily, Y. Jararweh, T. Baker, An edge computing based smart healthcare framework for resource management. Sensors. 18(12), 4307 (2018)

8. X. Lai, W. Zou, D. Xie, X. Li, L. Fan, Df relaying networks with randomly distributed interferers. IEEE Access. 5, 18909-18917 (2017)

9. Y. Zhang, C. Yin, W. Qilin, Q. He, H. Zhu, Location-aware deep collaborative filtering for service recommendation. IEEE Trans. Syst. Man Cybern. Syst. (2019). https://doi.org/10.1109/tsmc.2019.2931723

10. Y. Zhang, K. Wang, Q. He, F. Chen, S. Deng, Z. Zheng, Y. Yang, Covering-based web service quality prediction via neighborhood-aware matrix factorization. IEEE Trans. Serv. Comput. (2019). https://doi.org/10. $1109 /$ tsc.2019.2891517

11. G. Liu, X. Yan, Z. He, Y. Rao, J. Xia, L. Fan, Deep learning-based channel prediction for edge computing networks toward intelligent connected vehicles. IEEE Access. 7, 114487-114495 (2019)

12. X. Liu, J. Li, Z. Yang, J. Li, A task collaborative execution policy in mobile cloud computing. Chin. J. Comput. 41(3), 364-377 (2017)

13. M. Mukherjee, L. Shu, H. Likun, G. P. Hancke, C. Zhu, Sleep scheduling in industrial wireless sensor networks for toxic gas monitoring. IEEE Wirel. Commun. 24(4), 106-112 (2017)

14. E. Ahmed, I. Yaqoob, A. Gani, M. Imran, M. Guizani, Social-aware resource allocation and optimization for D2D communication. IEEE Wirel. Commun. 24(3), 122-129 (2017)

15. H. Wang, J. Gong, Y. Zhuang, H. Shen, J. L. Healthedge, in 2017 IEEE International Conference on Big Data (Big Data). Task scheduling for edge computing with health emergency and human behavior consideration in smart homes (IEEE, 2017), pp. 1213-1222. https://doi.org/10.1109/nas. 2017.8026861

16. Y. Zhang, G. Cui, S. Deng, F. Chen, Y. Wang, Q. He, Efficient query of quality correlation for service composition. IEEE Trans. Serv. Comput. (2018). https://doi.org/10.1109/tsc.2018.2830773

17. X. Fang, L. Liu, X. Liu, The analysis method about change region of process model based on module. Appl. Math. Inf. Sci. 8(1), 221-227 (2014)

18. N. Khan, M. G. Martini, Qoe-driven multi-user scheduling and rate adaptation with reduced cross-layer signaling for scalable video streaming over LTE wireless systems. EURASIP J. Wirel. Commun. Netw. 2016(1), 93 (2016)

19. T. P. C. de Andrade, C. A. Astudillo, N. L. S. da Fonseca, Allocation of control resources for machine-to-machine and human-to-human communications over Ite/lte-a networks. IEEE Internet Things J. 3(3), 366-377 (2016)

20. F. Liu, The performance evaluation analysis for human resource based on back propagation neural network. Technol. Dev. Enterp. 30(23), 123-133 (2011)

21. Y. Feng, The allocation of human resources in industrial network under virtual cooperation. Reformation Strateg. 29(1), 60-63 (2013) 
22. L. C. E. Silva, A. P. C. S. Costa, Decision model for allocating human resources in information system projects. Int. J. Project Manag. 31(1), 100-108 (2013)

23. C. L. Ji, D.-L. Zhao, B.-F. Ge, K.-W. Yang, Y.-W. Chen, A link prediction method for heterogeneous networks based on bp neural network. Phys. A Stat. Mech. Appl., S0378437117312645 (2017)

24. Z. Zhongwei, M. Hongyan, Z. Jun, J. Huiping, A rule extraction method based on multi-objective co-evolutionary genetic algorithm. J. Shandong Univ.(Eng. Sci.) 49(2), 122-130 (2019)

25. W. Liu, Z. Wang, X. Liu, N. Zeng, Y. Liu, F. E. Alsaadi, A survey of deep neural network architectures and their applications. Neurocomputing. 234, $11-26(2017)$

26. L. Qi, C. Yi, Y. Yuan, F. Shucun, X. Xiaolong, A QoS-aware virtual machine scheduling method for energy conservation in cloud-based cyber-physical systems. World Wide Web. 1(5), 997-1012 (2019)

27. M. Chen, V. C. M. Leung, From cloud-based communications to cognition-based communications: A computing perspective. Comput.Commun. 128, 74-79 (2018)

28. L. Qi, Q. He, F. Chen, W. Dou, S. Wan, X. Zhang, Xiaolong X, Finding all you need: Web APIs recommendation in web of things through keywords search. IEEE Trans. Comput. Soc. Syst. 20(1), 1063-1072 (2019)

29. X. Xu, Y. Chen, X. Zhang, Q. Liu, X. Liu, L. Qi, A blockchain-based computation offloading method for edge computing in $5 \mathrm{~g}$ networks. Softw. Pract. Experience (2019). https://doi.org/10.1002/spe.2749

30. K. Lin, C. Li, D. Tian, A. Ghoneim, M. Shamim Hossain, S. U. Amin, Artificial-intelligence-based data analytics for cognitive communication in heterogeneous wireless networks. IEEE Wirel. Commun. 26(3), 83-89 (2019)

31. X. Xu, Y. Li, T. Huang, Y. Xue, K. Peng, L. Qi, W. Dou, An energy-aware computation offloading method for smart edge computing in wireless metropolitan area networks. J. Netw. Comput. Appl. 133(1), 75-85 (2019)

32. X. Xu, X. Yuan, Q. Lianyong, Y. Yuan, Z. Xuyun, U. Tariq, W. Shaohua, An edge computing-enabled computation offloading method with privacy preservation for internet of connected vehicles. Future Gener. Comput. Syst. 96(1), 89-100 (2019)

33. H. Singh, A. Bhasin, P. K. Secure, Efficient resource scheduling by swarm in cloud computing. J. Discret. Math. Sci. Crypt. 22(2), 127-137 (2019)

34. X. Xu, L. Qingxiang, L. Yun, P. Kai, Z. Xuyun, M. Shunmei, Q. Lianyong, A computation offloading method over big data for loT-enabled cloud-edge computing. Future Gener. Comput. Syst. 95, 522-533 (2019)

35. V. Priya, C. Sathiya Kumar, R. Kannan, Resource scheduling algorithm with load balancing for cloud service provisioning. Appl. Soft Comput. 76, 416-424 (2019)

36. Y. Liang, X. Cao, D. He, C. Wang, W. Zhang, Z. Weixiong, in The 25th Internation Joint Conference on Artificial Intelligence. Modularity based community detection with deep learning (IJCAI, 2016), pp. 2252-2258

37. J. Cao, J. Di, L. Yang, J. Dang, Incorporating network structure with node contents for community detection on large networks using deep learning. Neurocomputing. 297, 71-81 (2018)

\section{Publisher's Note}

Springer Nature remains neutral with regard to jurisdictional claims in published maps and institutional affiliations.

\section{Submit your manuscript to a SpringerOpen ${ }^{\odot}$ journal and benefit from:}

- Convenient online submission

- Rigorous peer review

- Open access: articles freely available online

- High visibility within the field

- Retaining the copyright to your article

Submit your next manuscript at $\gg$ springeropen.com 